\title{
Ethanol-Induced Reorganization of the Liquid-Ordered Phase: Enhancement of Cholesterol-Phospholipid Association
}

\author{
Jianbing Zhang, Honghua Cao, Bingwen Jing, and Steven L. Regen * \\ Department of Chemistry, Lehigh University, Bethlehem, Pennsylvania 18015
}

\begin{abstract}
This paper records, what is believed to be, the first evidence for the reorganization of the liquidordered phase by ethanol. Specifically, ethanol has been found to significantly enhance sterolphospholipid association in liquid-ordered bilayers derived from 1,2-dipalmitoyl-sn-glycero-3phosphocholine (DPPC) plus cholesterol, and also 1,2-distearoyl-sn-glycero-3-phosphocholine (DSPC) plus cholesterol. The evidence for such reorganization comes from a series of nearestneighbor recognition (NNR) experiments that have been carried out, where low concentrations of equilibrating lipid dimers (i.e., "reporter molecules") have been used to detect changes in the phase composition of host membranes made from varying mixtures of DPPC/cholesterol, and also DSPC/ cholesterol, in the presence and in the absence of ethanol. These findings have important biological implications, which are briefly discussed.
\end{abstract}

\section{Introduction}

Ethanol is one of the most popular, yet one of the least understood, depressants that is currently in use. In particular, the mechanism by which this agent affects neurological function at the molecular level is not currently known. ${ }^{1-7}$ Although neural membranes appear to be altered in some way by the presence of ethanol, the exact nature of the interactions that take place among ethanol, membrane proteins and the lipid framework remains obscure.

Because of its lack of specificity, it has generally been assumed that ethanol's primary "target" in cell membranes is the lipid framework. ${ }^{8-10}$ For this reason, there has been considerable effort made to gain insight into how ethanol influences the structure and properties of lipid bilayers in model systems. Based on such studies, it is now clear that ethanol can induce a disordering of the acyl chains of fluid phospholipids, as well as the formation of an interdigitated gel phase..$^{3,11-17}$ It is also clear that the presence of high concentrations of cholesterol ( $\geq 30 \mathrm{~mol} \%$ ) greatly reduces ethanol's ability to disorder fluid phospholipids. ${ }^{3,18}$ From detailed NMR studies, it also appears that ethanol favors binding to lipid membranes near the lipid/water interface, in regions that lie close to the glycerol backbone and the "upper" segments of the hydrocarbon chains of the lipids. $3,16,19,20$

In the work reported herein, our primary aim was to clarify one fundamentally important issue regarding the interactions between ethanol and lipid membranes, which has not previously been addressed. Specifically, can ethanol alter the organization within the liquid-ordered phase? Given a growing body of evidence for the existence of liquid-ordered regions within cell membranes (i.e., regions where cholesterol associates with high-melting lipids), which lie in contact with liquid-disordered regions (i.e., regions comprised largely of low-melting glycerophospholipids), this question has considerable relevance to the biological action of 
ethanol. ${ }^{21-26}$ Thus, if ethanol were to alter cholesterol-phospholipid association, this could affect the structure, composition and function of liquid-ordered as well as liquid-disordered regions.

With this goal in mind, we have sought to quantify the effects of ethanol on sterol-phospholipid interactions in the liquid-ordered, as well as the liquid-disordered, phase. For this purpose, we have utilized the nearest-neighbor recognition (NNR) method, operating in a chemical-sensing mode. ${ }^{26,27}$ Specifically, we have used low concentrations of equilibrating lipid dimers (i.e., "reporter molecules") to detect changes in the phase composition of host membranes made from varying mixtures of cholesterol and 1,2-dipalrnitoyl-sn-glycero-3-phosphocholine (DPPC), and also varying mixtures of cholesterol plus 1,2-distearoyl-sn-glycero-3phosphocholine (DSPC) in the presence and in the absence of ethanol (Chart 1).

As discussed elsewhere, NNR measurements provide molecular-level snapshots of bilayer organization by detecting and quantifying the thermodynamic tendency of exchangeable monomers to become nearest-neighbors of one another. ${ }^{26,27}$ Typically, two lipids of interest $(\mathbf{A}$ and $\mathbf{B})$ are converted into exchangeable dimers $(\mathbf{A A}, \mathbf{A B}$ and $\mathbf{B B})$, which are then allowed to undergo monomer interchange via thiolate-disulfide interchange. These equilibria are then defined by a constant, $K$, which governs the monomer interchange among $\mathbf{A A}, \mathbf{B B}$ and $\mathbf{A B}$ (eqs 1 and 2). When monomers $\mathbf{A}$ and $\mathbf{B}$ mix ideally, this is reflected by an equilibrium constant that equals 4.0. When homo-associations are favored, the equilibrium constant is less than 4.0; favored hetero-associations are indicated by a value that is greater than 4.0.

$$
\begin{gathered}
\mathrm{AA}+\mathrm{BB} \rightarrow \underset{\text { equilibrium mixture }}{[\mathrm{AA}+\mathrm{AB}+\mathrm{BB}]} \leftarrow \mathrm{AB} \\
\mathrm{AA}+\mathrm{BB} \stackrel{K}{\rightleftharpoons} 2 \mathrm{AB} \\
K=[\mathrm{AB}]^{2} /([\mathrm{AA}][\mathrm{BB}])
\end{gathered}
$$

\section{Results and Discussion}

\section{Host Membranes}

Our choice of DPPC/cholesterol and DSPC/cholesterol as host membranes for the present study was based their broad use in model studies, and also the fact that their phase diagrams are wellestablished. The phase diagram for DPPC/cholesterol, in particular, has been extensively characterized by a combination of differential scanning calorimetry, $\mathrm{x}$-ray diffraction, ${ }^{2} \mathrm{H}$ NMR, and spin-label ESR measurements, along with statistical thermodynamic considerations. 28 Thus, at $60^{\circ} \mathrm{C}$, the liquid-disordered region extends from ca. $0-15 \mathrm{~mol} \%$ cholesterol, and the liquid-disordered/liquid-ordered coexistence region extends from ca. 15 to $30 \mathrm{~mol} \%$ cholesterol. At higher sterol concentrations ( $>30 \mathrm{~mol} \%$ ), the membrane is fully converted into the liquid-ordered phase. Although the phase diagram of DSPC/cholesterol has been investigated to a lesser extent, ESR measurements indicate that at $60^{\circ} \mathrm{C}$, the liquid-disordered and liquid-disordered/liquid-ordered coexistence regions extend from ca. 0-8 mol\% cholesterol and ca. $8-27$ mol\% cholesterol, respectively. ${ }^{29}$ Beyond $27 \%$ cholesterol, the membrane is fully converted to the liquid-ordered phase. In the present work, all NNR experiments have been carried out at $60^{\circ} \mathrm{C}$, because the liquid-disordered phase, the liquiddisordered/liquid-ordered coexistence region, and the liquid-ordered phase in DPPC/ cholesterol and DSPC/cholesterol systems are accessible by varying the cholesterol content within these membranes. 


\section{NNR Reporter Molecules}

The exchangeable lipids that were chosen as reporter molecules for this investigation (i.e., $\mathbf{1}$, 2 and 3) bear the same diacylglycerol and sterol frameworks as DPPC, cholesterol and DSPC, respectively (Chart 2). It is noteworthy, in this regard, that despite the presence of a disulfide bridge in the head group region, and despite the presence of a net negative charge in the phospholipids, the mixing properties of such lipids bear a striking resemblance to those of cholesterol and the corresponding phosphatidylcholines. That this is the case is evident from the fact that the mixing behavior of 1 with 2 (i.e., the value of $K$ ) in host membranes made from DPPC/cholesterol has a similar dependency on the total sterol content, as compared with undiluted bilayers made exclusively from $\mathbf{1}$ and 2.26,30 Thus, in both of these systems, $\mathbf{1}$ and 2 have been found to (i) mix nearly ideally at low sterol concentrations ( $\leq 15 \mathrm{~mol} \%$ ), (ii) favor the formation of heterodimer at higher sterol concentrations, and (iii) exhibit a limiting value of $K$ that is equal to ca. 5.4 when fully converted to the liquid-ordered phase. This similarity implies that the lipid microenvironment, which defines $K$, is similar in both membranes. It also implies that the lateral arrangement of neighboring DPPC and cholesterol molecules is similar to that of neighboring $\mathbf{1}$ and $\mathbf{2}$. In other words, the mixing of DPPC with cholesterol must be similar to the mixing of $\mathbf{1}$ with $\mathbf{2}$. It also means that $\mathbf{1}$ and $\mathbf{2}$ have become an integral part of host membranes made from DPPC and cholesterol; that is, the exchangeable and nonexchangeable lipids are well-mixed. That the presence of a disulfide bridge does not, in any way, influence the packing properties of such lipids (i.e., their alignment with neighboring lipids) has already been established through monolayer measurements, where limiting areas and condensing properties have been found to be indistinguishable from lipid analogs that are devoid of the such a bridge. ${ }^{30}$ In addition, the close similarity in the packing properties between these exchangeable and nonexchangeable lipids is also evident from their nearly identical gel to liquid-crystalline phase transition temperatures $\left(T_{\mathrm{m}}\right)$, which are: $41.9^{\circ}, 41.5^{\circ}, 55.4^{\circ}$ and $54.3^{\circ} \mathrm{C}$ for $\{\mathbf{1}, \mathbf{1}\}$, DPPC, $\{\mathbf{3}, \mathbf{3}\}$ and DSPC, respectively. 31

\section{Influence of Ethanol on the Mixing of 1 with 2 in DPPC/Cholesterol Host Membranes}

To gain insight into the effect that ethanol has on sterol-phospholipid mixing, we first carried out a series of NNR experiments using liposomes that were made from $5 \mathrm{~mol} \%$ of an equimolar mixture of $\mathbf{1}$ and $\mathbf{2}$ plus $95 \mathrm{~mol} \%$ of varying ratios of DPPC/cholesterol. For this purpose, two independent sets of experiments were performed to ensure that equilibrium product mixtures were obtained. Thus, in one set of experiments, pure heterodimer $\{\mathbf{1 , 2}\}$ was used as the source of these exchangeable lipids. In a second set, we used an equimolar mixture of the corresponding homodimers, $\{\mathbf{1}, \mathbf{1}\}$ and $\{\mathbf{2 , 2}\}$. Both dispersions were made using an aqueous buffer that contained $5 \%$ ethanol (v/v). Analysis after extrusion, using standardized conditions $\left(200 \mathrm{~nm}\right.$ Nuclepore membranes, $60^{\circ} \mathrm{C}$ ), revealed a partial lowering of the ethanol concentration to $2.9 \%(\mathrm{v} / \mathrm{v})$, due to evaporation (see Supporting Information). Dynamic light scattering showed an average liposome diameter of $200 \mathrm{~nm}$. Thiolate-disulfide interchange reactions were then carried out at $60^{\circ} \mathrm{C}$ using experimental procedures similar to those previously described.

26 Values of $K$ were calculated from the average dimer composition from both sets of experiments, where product mixtures were analyzed at six different time intervals. Thus, 12 sets of convergent data were used to obtain each value of $K$. Examination of these dispersions at the end of the reaction by dynamic light scattering showed no change in the particle size or size distribution.

A plot of $K$ as a function of the total sterol content is presented in Figure 1. Also shown in this figure are results that were previously obtained from NNR experiments carried out in the absence of ethanol. ${ }^{26} \mathrm{~A}$ linear regression analysis of the data in the region where $K$ increases with mol \% sterol, together with horizontal segments, yield "take-off" points (the mol \% sterol where $K$ starts to increase with increasing sterol concentrations, corresponding to the onset of the liquid-ordered phase) of 12.2 and $8.9 \mathrm{~mol} \%$ sterol, in the absence and in the presence of 
ethanol, respectively. At high sterol concentrations, ethanol significantly increased $K$; the maximum effect being observed when the host membrane was fully converted into the liquidordered phase ( $\geq 30 \mathrm{~mol} \%$ sterol). ${ }^{32}$ The linear increase in $K$ with increasing mole percentages of sterol implies that the conversion from the liquid-disordered phase to the liquid-ordered phase is also linear. Given the magnitude of the change in free energy for the mixing between 1 and $\mathbf{2}$, on going from the liquid-disordered to the liquid-ordered phase (i.e., 560 joule/mole of exchangeable lipid), ethanol's effect in altering the lateral arrangement of $\mathbf{1}$ and $\mathbf{2}$ in the liquid-ordered phase (298 joule/mol of exchangeable lipid) is very significant. ${ }^{33}$ The apparent shift of the take-off points to lower sterol concentrations in the presence of ethanol is fully consistent with the effects observed at higher sterol concentrations. Thus, stronger sterolphospholipid association is expected to result in the liquid-ordered phase being formed at a reduced sterol concentration. The fact that $30 \mathrm{~mol} \%$ remains as the point of discontinuity in the presence of alcohol further indicates that these NNR experiments are still measuring the amount of liquid-ordered phase that exists within the membrane.

\section{Influence of Ethanol on the Mixing of 2 with 3 in DSPC/Cholesterol Host Membranes}

An analogous series of NNR experiments was then performed in DSPC/cholesterol host membranes using $\mathbf{2}$ and $\mathbf{3}$ as the chemical sensor (Figure 2). Qualitatively, the results that were obtained were similar to those shown in Figure 1. In this case, however, the take-off points were shifted to ca. 8.6 and $5.2 \mathrm{~mol} \%$ of sterol in the absence and in the presence of ethanol, respectively. In addition, the maximum value in $K$ was increased from ca. 5.4 to 5.7 (absence of ethanol), and from 6.7 to 7.3 (in the presence of ethanol). These higher $K$ values are a likely consequence of stronger hydrophobic interactions between the longer, higher melting phospholipids and neighboring sterols in the form of a "condensed complex". 24

\section{Influence of Ethanol on the Mixing of 2 and 3 in DPPC/Cholesterol Host Membranes}

In a third series of NNR experiments, we measured the mixing of $\mathbf{2}$ with $\mathbf{3}$ in DPPC/cholesterol host membranes. In principle, such experiments can provide insight into whether the observed enhancement in sterol-phospholipid association by ethanol is confined to the reporter molecules, themselves, or if it extends to the host lipids.

Figure 3 shows the values of $K$ that were observed for the mixing of $\mathbf{2}$ and $\mathbf{3}$ in host membranes made from DPPC/cholesterol as a function of sterol content in the absence, and in the presence, of ethanol. If one compares the take-off points for these systems, they are clearly much closer to those found in Figure 1 than those shown in Figure 2. Since the host membranes that were used to generate Figures 1 and 3 were the same (only the reporter molecules differ), these results indicate that it is ethanol's effect on the host lipids that is largely responsible for its effect on $K$. In other words, ethanol enhances the association between DPPC and cholesterol, which, in turn, provides a microenvironment that enhances the association between $\mathbf{2}$ and $\mathbf{3}$. The fact that the $K$ values in Figure 3 are intermediate between those found in Figures 1 and 2 further indicates that these exchangeable lipids contribute to the overall strength of the "condensed complexes" that are formed with the nonexchangeable host lipids.

To obtain evidence that the interactions between ethanol and the exchangeable lipids are similar to the interactions between ethanol and the host lipids, we carried out one final NNR experiment using an ethanol-containing dispersion that was made, exclusively, from $65 \mathrm{~mol} \% 1$ and 35 mol\% 2. Since $K$ is controlled by the lipid microenvironment surrounding $\mathbf{1}$ and $\mathbf{2}$, if the effect of ethanol on the microenvironment that is created by neighboring $\mathbf{1}$ and $\mathbf{2}$ were different from the one that is created by neighboring DPPC and cholesterol, then $K$ should be different. The fact that the value of $K(6.7)$ was found to be the same as that found in DPPC/cholesterol host membranes containing $35 \mathrm{~mol} \%$ sterol (Figure 1) indicates, therefore, that ethanol is enhancing 
DPPC - cholesterol association in a way that is very similar to how it enhances the association between $\mathbf{1}$ and $\mathbf{2}$.

\section{Proposed Mechanism of Biological Action}

Based on ethanol's ability to stabilize sterol-phospholipid association in the liquid-ordered phase, we postulate that ethanol exerts a similar effect on neural cell membranes. Specifically, we posit that ethanol's primary mode of action is to "tie up" cholesterol and high-melting phospholipids in the liquid-ordered phase, which results in a reduction in the quantity of each lipid type that is available for associating with transmembrane proteins in liquid-ordered as well as liquid-disordered regions of neural membranes. We also posit that such lipid-protein association is essential for maintaining the proper structure and functioning of these proteins. It is noteworthy, in this regard, that although integral membrane proteins appear to favor liquid-disordered regions, evidence has begun to emerge indicating that certain ion channel-forming proteins strongly favor cholesterol-rich regions of cell membranes. ${ }^{34,35} \mathrm{In}$ preliminary studies, we have found that ethanol shows a similar effect on liquid-ordered bilayers that contain a transmembrane peptide; that is, in a model system that more closely mimics neural cell membranes. Thus, for DPPC/cholesterol bilayers that contain $5 \mathrm{~mol} \%$ of the non-channel form of gramicidin A ( $n c-\mathrm{gA})$ and $30 \mathrm{~mol} \%$ total sterol, the $K$ value for the mixing of 1 and 2 was found to increase from $4.54 \pm 0.12$ to $5.34 \pm 0.12$, on going from 0 to $2.9 \%$ ethanol at $60^{\circ} \mathrm{C} .{ }^{36-38}$

The underlying mechanism by which ethanol enhances sterol-phospholipid association remains to be established. One possibility is that it may be acting as a "filler". Specifically, since the geometry of cholesterol cannot perfectly match that of a neighboring phospholipid, some hydrocarbon gaps between them are expected. Thus, by inserting into these gaps and replacing water molecules near the membrane-water interface, ethanol may enhance hydrocarbon contact between these two very different types of lipids. Efforts aimed at gaining deeper insight into the action of ethanol and other depressants on membrane organization are continuing in our laboratory.

\section{Supplementary Material}

Refer to Web version on PubMed Central for supplementary material.

\section{Acknowledgements}

We are grateful to the National Institutes of Health (PHS GM56149) for support of this research.

\section{References}

1. Chin JH, Goldsterin DB. Molecular Pharmacol 1981;19:425-431.

2. Chin JH, Goldstein DB. Mol Pharmacol 1977;13:435-441. [PubMed: 876032]

3. Barry JA, Gawrisch K. Biochemistry 1995;34:8852-8860. [PubMed: 7612626]

4. Wood WG, Schroeder F. Life Sci 1988;43:467-475. [PubMed: 3043131]

5. Trandum C, Westh P, Jorgensen K, Mouritsen OG. Biophys J 2000;78:2486-2492. [PubMed: 10777745]

6. Trandum C, Westh P, Jorgensen K, Mouritsen OG. J Phys Chem 1999;103:4751-4756.

7. Komatsu H, Guy PT, Rowe ES. Chem Phys Lipids 1993;65:11-21. [PubMed: 8348673]

8. Goldstein DB. Ann Rev Pharmacol Toxicol 1984;24:43-64. [PubMed: 6329077]

9. Goldstein DB. Annals of Emergency Medicine 1986;15:43/1013-48/1048.

10. Sun GY, Sun AY. Alcoholism: Clinical and Experimental Research 1985;9:164-179.

11. Rowe ES, Cutrera TA. Biochemistry 1990;29:10398-10404. [PubMed: 2261481] 
12. Nambi P, Rowe ES, McIntosh TJ. Biochemistry 1988;27:9175-9182. [PubMed: 3242621]

13. Komatsu H, Rowe ES. Biochemistry 1991;30:2463-2470. [PubMed: 2001373]

14. Mou J, Yang J, Huang C, Shao Z. Biochemistry 1994;33:9981-9985. [PubMed: 8061008]

15. McClain RL, Breen JJ. Langmuir 2001;17:5121-5124.

16. Barry JA, Gawrisch K. Biochemistry 1994;33:8082-8088. [PubMed: 8025114]

17. Wachtel E, Borochov N, Bach D, Miller IR. Chem Phys Lipids 1998;92:127-137. [PubMed: 9682467]

18. Bach D, Borochov N, Wachtel E. Chem Phys Lipids 2002;114:123-130. [PubMed: 11934393]

19. Hole LL, Gawrisch K. Biochemistry 1997;36:4669-4674. [PubMed: 9109678]

20. Feller SE, Brown CA, Nizza DT, Gawrisch K. Biophys J 2002;82:1396-1404. [PubMed: 11867455]

21. Edidin M. Annu Rev Biomol Struct 2003;32:257-283.

22. Simons K, Vaz WLC. Annu Rev Biomol Struct 2004;33:269-295.

23. Simons K, Toomre D. Natl Rev Mol Cell Biol 2000;1:31-39.

24. McConnell HM, Radhakrishnan A. Biochim Biophys Acta 2003;1610:159-173. [PubMed: 12648771]

25. Sugahara M, Uragami M, Regen SL. J Am Chem Soc 2003;125:13040-13041. [PubMed: 14570473]

26. Cao H, Zhang J, Jing B, Regen SL. J Am Chem Soc 2005;127:8813-8816. [PubMed: 15954788]

27. For a review of the NNR method, see: (a) Davidson SKM, Regen SL. Chem Rev 1997;97:1269-1280. [PubMed: 11851451] (b) Cao H, Tokutake N, Regen SL. J Am Chem Soc 2003;125:16182-16183. [PubMed: 14692747] (c) Sugahara M, Uragami M, Regen SL. J Am Chem Soc 2002;124:4253-4256. [PubMed: 11960454]

28. Sankaram MB, Thompson TE. Proc Natl Acad Sci, USA 1991;88:8686-8690. [PubMed: 1656453]

29. Almeida PF, Vaz WCC, Thompson TE. Biophys J 1993;64:399-412. [PubMed: 8457666]

30. Sugahara M, Uragami M, Yan X, Regen SL. J Am Chem Soc 2001;123:7939-7940. [PubMed: 11493081]

31. Krisovitch SM, Regen SL. J Am Chem Soc 1992;114:9828-9835.

32. Similar NNR experiments that were carried out, in which the sterol content was held constant at 30 mol \% and the concentration of ethanol that was used to prepare the liposomes was increased to $10 \%$ (v/v) gave a $K$ value that was the same as that found using $5 \%$ ethanol. When the concentration of ethanol was lowered to $1 \%$, however, the $K$ value was the same as that found in its absence. Using 1 and $10 \%$ ethanol to form the vesicles under standardized conditions, the final ethanol concentrations in the dispersions were 0.53 and $5.25 \%$, respectively. In an attempt to estimate the mole fraction of ethanol that is present in the membrane bilayer, we incubated a multilamellar dispersion made from DPPC/cholesterol $(7 / 3, \mathrm{~mol} / \mathrm{mol})$ with an aqueous $\left(\mathrm{D}_{2} \mathrm{O}\right)$ solution containing $3.0 \%$ ethanol $(\mathrm{v} / \mathrm{v})$ [i.e., $75.6 \mathrm{mg}$ of total lipid plus $1.0 \mathrm{~mL}$ of solution] for $30 \mathrm{~min}$ at $60^{\circ} \mathrm{C}$, before and after centrifugation. Subsequent analysis of the solution phase by ${ }^{1} \mathrm{H}$ NMR indicated less than 13 ethanol molecules were taken up per phospholipid. Based on partition coefficients that have been previously estimated for analogous membranes made from 1,2-dimyristoyl-sn-glycero-3-phosphocholine (DMPC) and cholesterol by isothermal titration calorimetry, one can estimate that ca. 10-50 ethanol molecules are being taken up per 100 phospholipids. ${ }^{5}$

33. NNR measurements that were carried out using $30 \mathrm{~mol} \%$ sterol, in the absence and in the presence of $2.9 \%$ ethanol, showed no variation in $K$ when the $\mathrm{NaCl}$ concentration in the buffer was varied from 100 to $300 \mathrm{mM}$. These results indicate that electrostatic effects do not contribute significantly to the observed mixing of $\mathbf{1}$ with $\mathbf{2}$.

34. Vidal A, McIntosh TJ. Biophys J 2005;89:1102-1108. [PubMed: 15908585]

35. Romanenko VG, Fang Y, Byfield F, Travis AJ, Vandenberg CA, Rothblat GH, Levitan I. Biophys J 2004;87:3850-3861. [PubMed: 15465867]

36. Results from previous NNR studies have led to the suggestion that cholesterol can associate with $n c$-gA to form complexes. ${ }^{37,38}$ The lower value of $K$ for $\mathbf{1}$ and $\mathbf{2}$, in the presence of $n c$-gA is consistent with this hypothesis, since it would reduce the amount of sterol that is available for associating with the phospholipid.

37. Sugahara M, Regen SL. Langmuir 2001;17:4413-4415.

38. Kishihara K, Jing B, Regen SL. Langmuir 2002;18:9635-9637. 


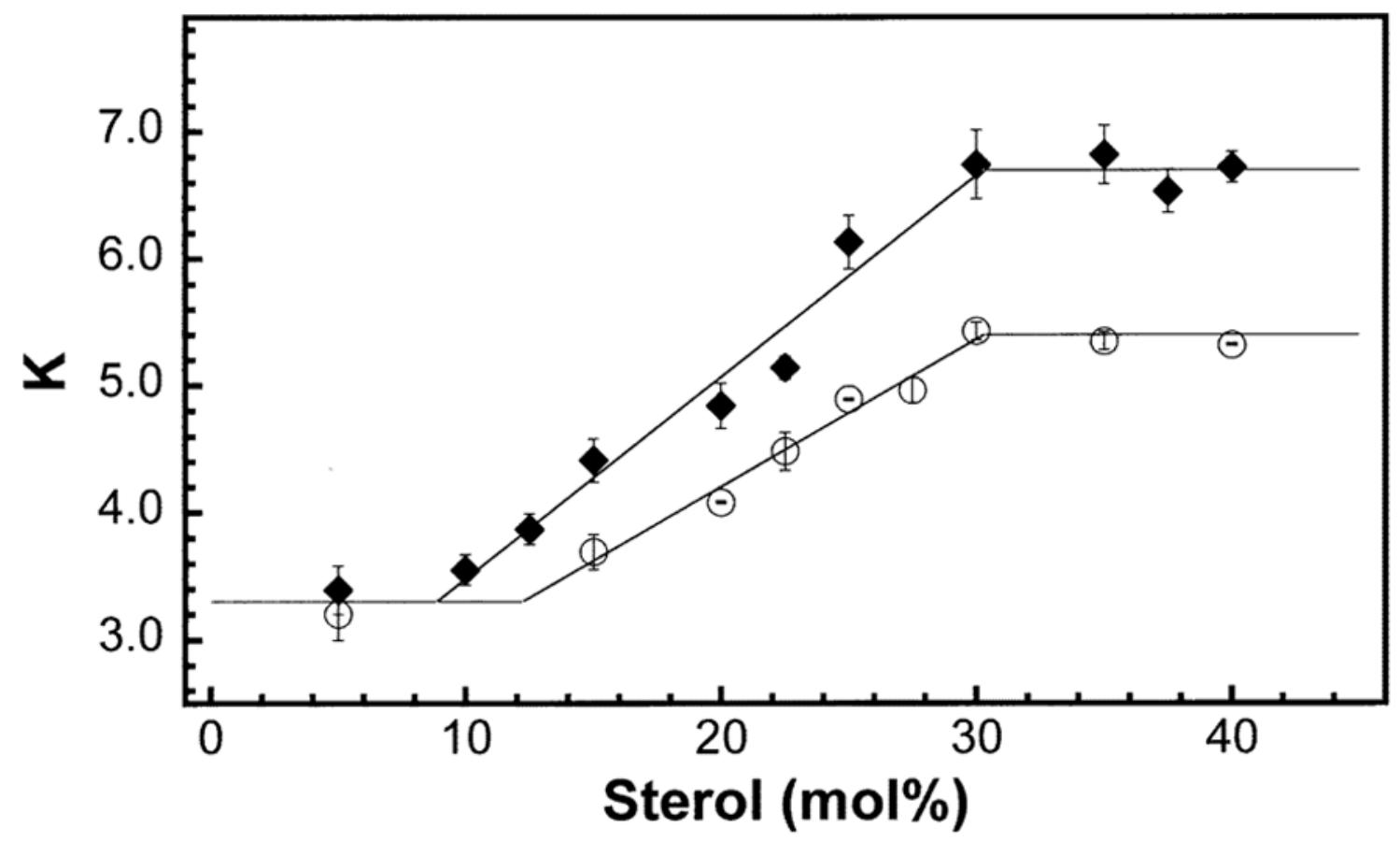

Figure 1.

Plot of $K\left(60^{\circ} \mathrm{C}\right)$ versus the mol \% of total sterol present in bilayers made from $95 \mathrm{~mol} \%$ of DPPC plus varying concentrations of cholesterol, plus $5 \mathrm{~mol} \%$ of exchangeable lipids (2.5 mol $\% 1$ plus 2.5 mol\% 2) in (०) buffer and ( ) buffer containing $2.9 \%$ ethanol (v/v). The solid lines that have been drawn in the intermediate regions (i.e., where $K$ increases with sterol content) are based on a linear regression analysis of the data according to: $y=0.116 x+1.88$ (absence of ethanol), and $y=0.159 x+1.89$ (presence of ethanol). In all cases, equilibrium was reached within $2 \mathrm{~h}$. The data that are shown in the absence in ethanol were taken from reference 26 


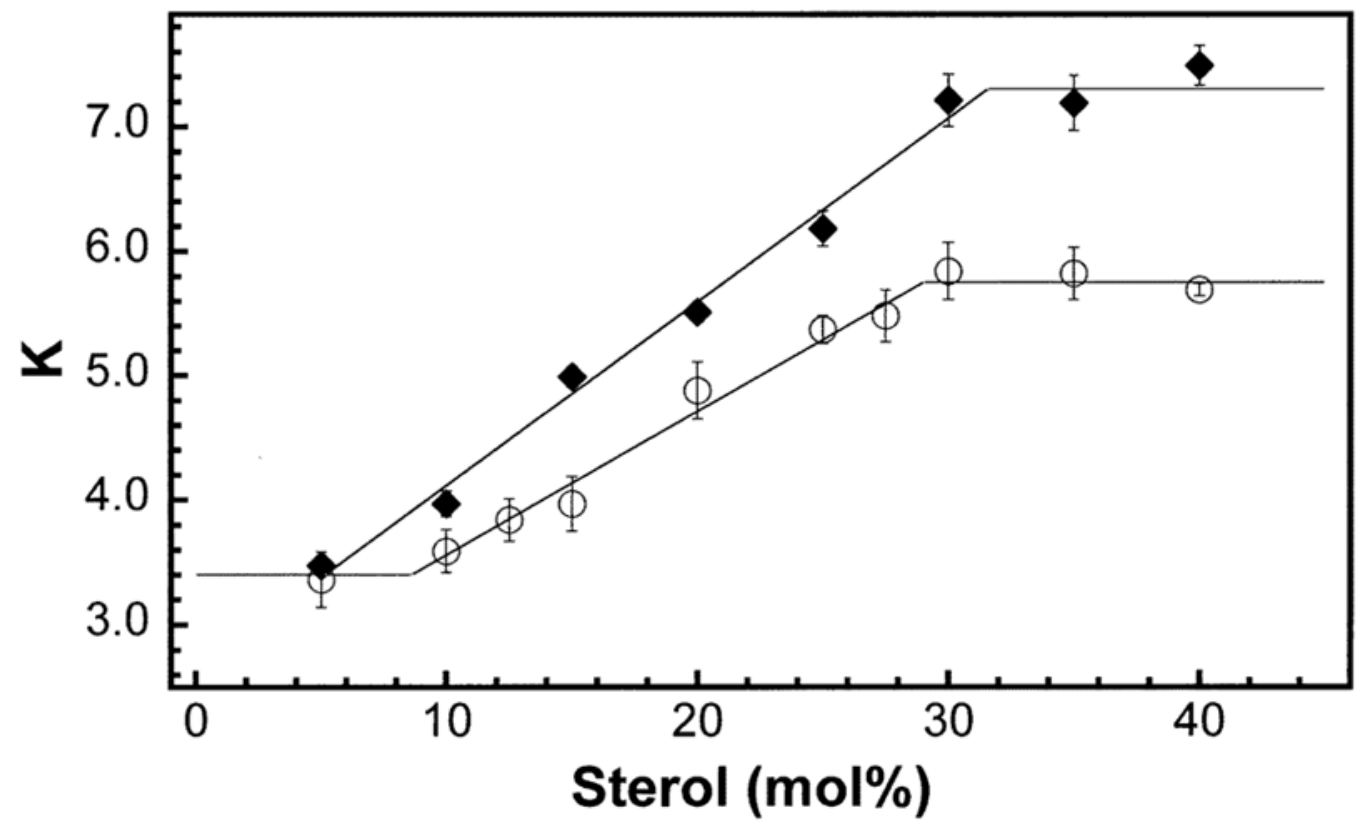

Figure 2.

Plot of $K\left(60^{\circ} \mathrm{C}\right)$ versus the mol \% of total sterol present in bilayers made from $95 \mathrm{~mol} \%$ of DSPC plus varying concentrations of cholesterol, plus $5 \mathrm{~mol} \%$ of exchangeable lipids ( $2.5 \mathrm{~mol}$ $\% 2$ plus $2.5 \mathrm{~mol} \% 3$ ) in (o) buffer and $(\diamond)$ buffer containing $2.9 \%$ ethanol (v/v). The solid lines that have been drawn in the intermediate regions (i.e., where $K$ increases with sterol content) are based on a linear regression analysis of the data according to: $y=0.115 x+2.40$ (absence of ethanol), and $y=0.148 x+2.64$ (presence of ethanol). In all cases, equilibrium was reached within $2 \mathrm{~h}$. The data that are shown in the absence of ethanol were taken from reference 26 


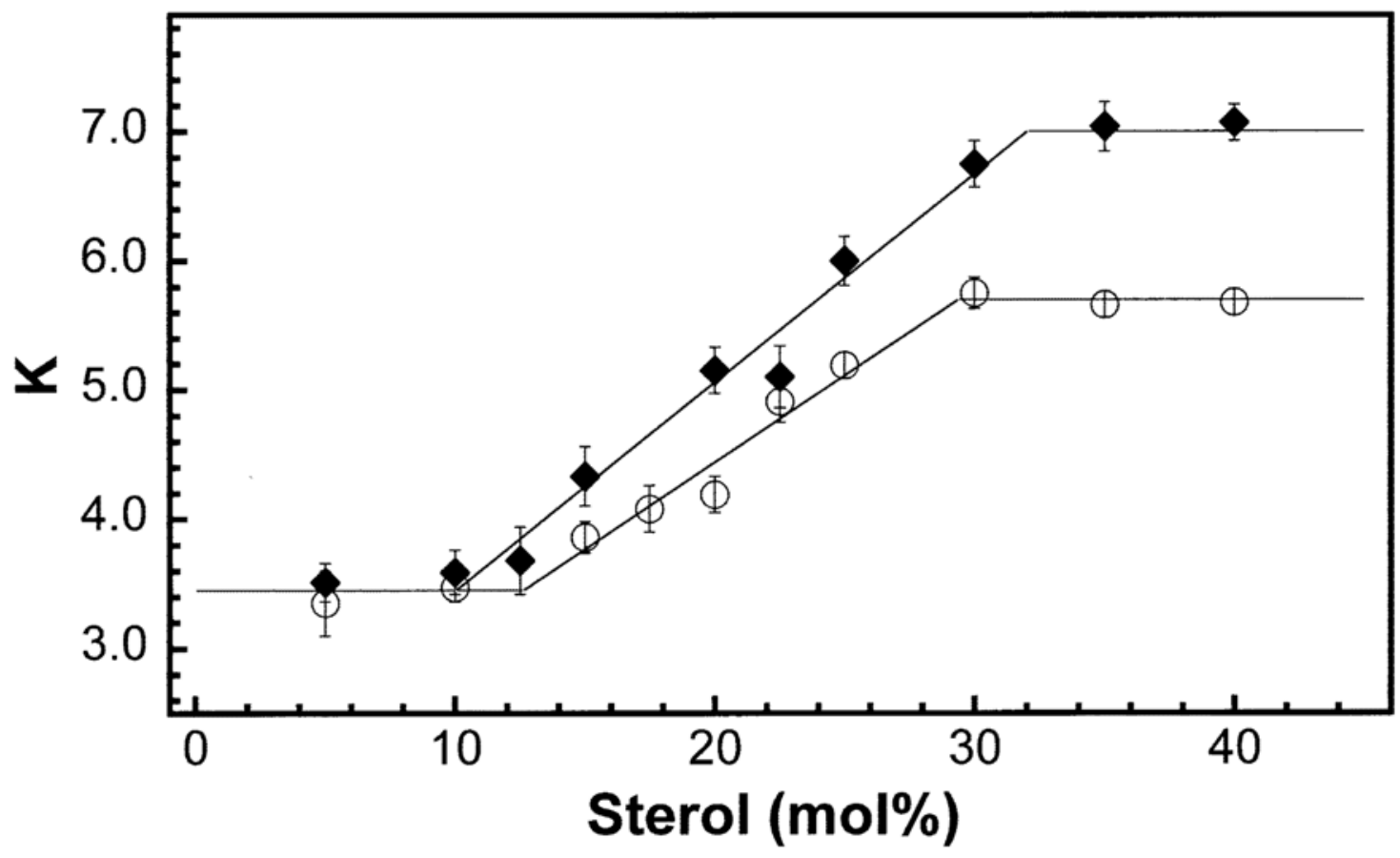

Figure 3.

Plot of $K\left(60^{\circ} \mathrm{C}\right)$ versus the mol \% of total sterol present in bilayers made from $95 \mathrm{~mol} \%$ of DPPC plus varying concentrations of cholesterol, plus $5 \mathrm{~mol} \%$ of exchangeable lipids (2.5 mol $\% 2$ plus $2.5 \mathrm{~mol} \% 3$ ) in (०) buffer and ( ) buffer containing $2.9 \%$ ethanol (v/v). The solid lines that have been drawn in the intermediate regions (i.e., where $K$ increases with sterol content) are based on a linear regression analysis of the data according to: $y=0.134 x+1.75$ (absence of ethanol), and $y=0.161 x+1.83$ (presence of ethanol). In all cases, equilibrium was reached within $2 \mathrm{~h}$. 


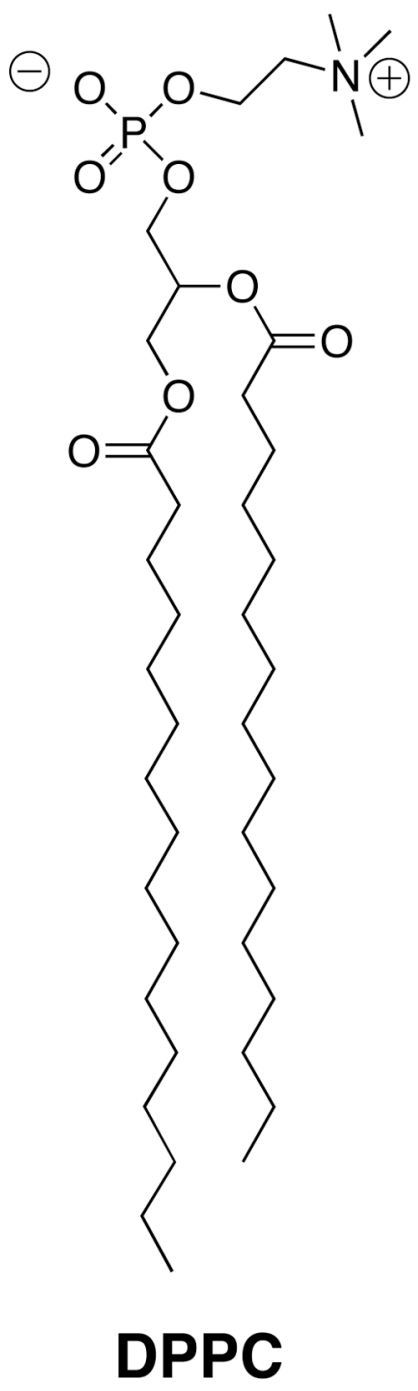

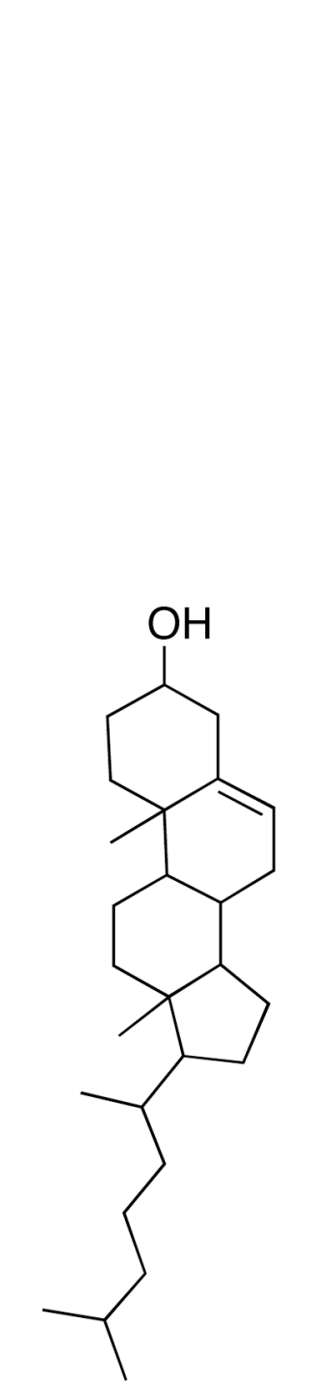

Cholesterol $\ominus$<smiles>COP(=O)(O)OCC[N+](C)(C)C</smiles>

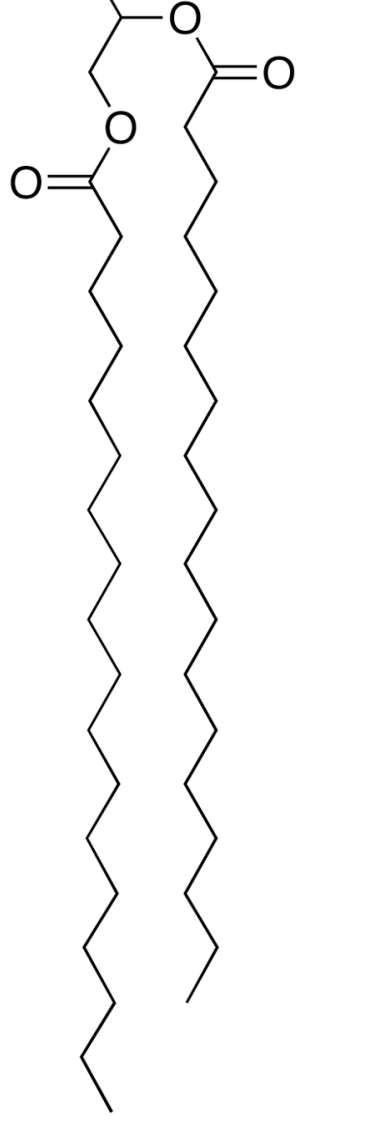

DSPC

Chart 1. 


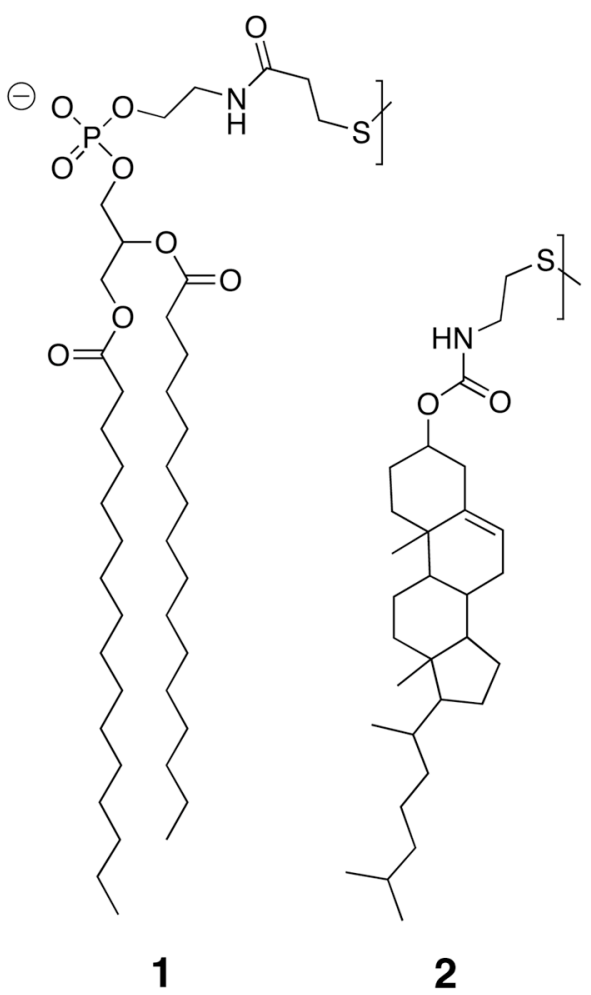<smiles>CC(C)CCC(=O)NCCOP(=O)([O-])OCC(C)O</smiles>

Chart 2. 